\title{
Reader Reading Promotion Based on Social Reading Theory
}

\author{
Xiufen Wang ${ }^{1,}$ a , Dongqing Chen ${ }^{2, b}$ \\ 1. Qingdao Technical College, Qingdao, China \\ 2.Qingdao Library, Qingdao, China \\ a.16910938@qq.com, b.252997580@qq.com
}

\begin{abstract}
Keywords: Social reading platform users; new media; statistical analysis method.
\end{abstract}
\begin{abstract}
To promote readers' reading properly, with the new media and the social reading platform users as the research object, the usage and the basic characteristics of the users were explored. Then, the use motivation, use attitude and use behavior of the social reading platform users were analyzed, and the relationship between the three was quantified. Based on the motivation demand theory, the use and gratification theory and the technology acceptance model, the literature analysis, in-depth interview, questionnaire survey and statistical analysis were adopted. The experiment results proved that the results obtained by the statistical analysis method were more objective. It is concluded that this study is intended to provide some reference for the development of the domestic social reading platform and to enrich the audience research in the communication of new media.
\end{abstract}

\section{Introduction}

On April 18, 2013, the 10th National Reading Survey of China was announced. The survey found that in 2012, the contact rate of national digital reading methods (online reading, mobile reading, e-reader reading, CD reading, PAD / MP4 / MPS reading) of 18-70 years old in China was 40.3\%, up $1.7 \%$ from 2011. In addition, in emerging media, the user's reading time through mobile phones shows an increasing trend, and the length of time spent on the Internet and the length of e-reader contact have declined. This shows that people's reading habits [1] and reading methods are changing. With the development of Internet technology and mobile Internet technology, people gradually shift from traditional reading to digital reading and intelligent reading. A variety of digital terminals have gradually replaced some of the paper products. The way of reading is also changing from the real to the virtual, from book and newspaper reading to screen reading.

At the same time, the emergence and rapid development of social network have liberated people from a closed reading situation and entered an era of socialized reading on an open platform. Therefore, the reading habits and reading status of reader have completely subverted by social media. User generated content (UGC), interactive sharing and reading will lead the new trend of future digital reading development. However, the proliferation of content has led to a deepening explosion and fragmentation of information and an increase in redundant information. It costs more time for users to get the information they need on the Internet. The increase in information noise and reading costs plagued users with reading information. Therefore, people began to research and develop information aggregation, classification, screening and recommendation techniques to solve the problems in reading process [2].

The first is the really simple syndication (RSS) [3]. It is also known as aggregated content, appearing on online news channels, blogs and wiki. The use of RSS subscription can quickly get information, and the website provides RSS output to allow users to get the latest updates of the content of the web site. At the same time, users can also read the content of the website that supports RSS output on the client side by means of an RSS-based aggregation tool software without opening the website content page. This technique has two major advantages: one is to aggregate a large amount of content, and the other is to create the conditions for offline reading. Today's RSS, users can also easily classify all information groups, add labels, label unread content, and share them into other social media. Secondly, personalized recommendation technology comes into being, which includes 
collaborative filtering, social recommendation and means that use a variety of data mining. Based on a certain amount of data, the site can analyze the user's interest, then recommend to the user targeted reading content according to their different interest needs.

Under the three aspects of the external environment, user needs and technology maturity, the social reading platform emerges as the times require. This new reading platform is user-centered and emphasizes the interaction, sharing, communication, social network and mobile reading. Personalized design and humanized service have changed people's reading habits, and they also have a profound impact on publishing industry, print media development, reading effectiveness and so on. This new reading platform reduces information noise and presents in a beautiful format. Meanwhile it has social advantages and personalized settings. Therefore, there is the birth and development of Flipboard.

\section{Research Status of Social Reading Platform}

\subsection{Domestic Research}

There is a lot of research on social networking sites in the academia and the industry, and there are few studies on the socialized reading platform. The author used social reading and personalized reading to search for keywords in CNKI [4] and Google academic database, and totally screened 30 articles. From the literature, it is found that both the academics and the industry have a good attitude towards this new Internet product. The related research perspectives are as follows:

The introductory description of socialized reading products: The characteristics and functional applications of the recommended description of socialized reading products are briefly introduced. The author concluded that the social literature reading platform has the following characteristics:

First, the user chose to read the content independently and complete the change from "what to read" to "I read what I want". Second, it is necessary to trace user's reading behavior and calculate user's interest, thus recommending the content they are interested. Third, the social reading platform is an interactive platform with personalized color. That is, the user can bind the account with some social networking sites, and the user can share the reading experience at any time in the reading process. At the same time, they pay attention to and review the comments. This is the most fascinating part of the social reading platform [5]. It is not simply a communication between human-machine, but also a communication between authors-readers and readers-readers. Qu Chenchen [6] does a research about the characteristics and advantages of the mobile Internet environment of digital reading. He believes that the mobile Internet expands the space for reading and shows the functions and patterns that the traditional Internet is difficult to display according to the mobile and phone's features. For example, the socialized reading platform integrates human socialized relations into reading, extending the space of the Internet and mobile Internet.

An analytical description of socialized reading products: In the analytical description of socialized reading products, the researchers made a deeper analysis on the basis of the functional introduction [7]. They believe that social media has broken the control of information in traditional media, changed the relationship between communicators and recipients, and constructed a trend of centralization and fragmentation. On the contrary, the social reading platform has led to another trend to a certain extent. The fragmented content will be aggregated again, using advanced computing and recommendation technology as the gatekeeper of users. This has laid the foundation for the later scholars to study the social reading platform. However, it has not explored the operation of the social reading platform, the industry chain and the profit mode.

\subsection{Foreign Research}

The United States is the first to focus on the development of the social reading platform. At present, the foreign reading platform is Flipboard and Zite. In 2010, Flipboard was named "the annual best iPad application". At the same time, the Time believes that Flipboard has triggered a social change. Then, it is regarded as one of the 50 major inventions in 2010. As the most powerful competitor of Flipboard, Zite has deep excavation of information discovery and personalization functions instead of imitating Flipboard's information processing mode of upgraded version RSS. 
For example, in the article "A personalized iPad magazine that learns what you like", Davar, Zite's CEO, introduces its development and functional characteristics. The biggest problem for readers is that in the face of massive content, they cannot obtain the personal information they need. However, Zite analyzes readers' interest in reading by capturing readers' browsing traces in the mainstream media, blogs and internet, and then provides users with reading content that suits their preferences. Davar points out that Zite is different from other competitors because it has more sources and has the function to automatically analyze user preferences. Zite [8] is also called "the smartest magazine on iPad" by Mashable. The article also quotes the Wall Street journal's comparative comments on Zite and Flipboard. Zite is not as flexible as Flipboard but contains some interesting content exploration innovation. If users want to handle a lot of information intelligently and provide APP with the content that they are interested in reading, it must be Zite. Davar also said that Zite will launch ads in the next few months because it is in the early stages of development and has no advertising content. In addition, Davar also proposes to work with publishers to aggregate their content to the Zite platform.

\section{Survey and Analysis of User Behavior on Social Reading Platform}

\subsection{Descriptive Analysis of Sample Demographics}

722 valid questionnaires were obtained for this survey $(\mathrm{N}=723)$. Therefore, the percentage base is confirmed by 723 empirical studies used by many media. The demographic variables have a greater impact on user motivation, attitudes and behaviors. Therefore, this article will conduct a detailed analysis. The detailed analysis is as follows:

The proportion of male users in the sample is slightly higher than the women: Among the 720 respondents who participated in the survey, 380 were men, accounting for $52.8 \%$ of the total number, while 340 were women, accounting for $47.2 \%$ of the total number, and $5.6 \%$ for male users. As shown in figure 1 , the proportion of male users is $5.6 \%$ higher than the women. This suggests that male users have a slightly higher reading demand than women.

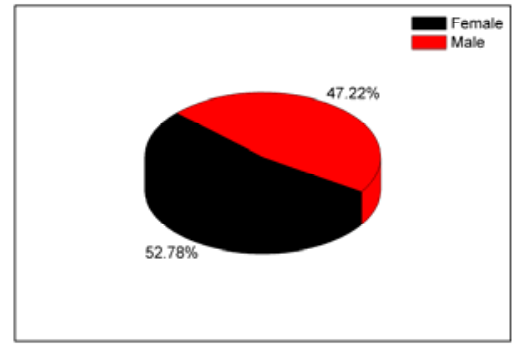

Figure. 1. Social reading platform user gender structure diagram

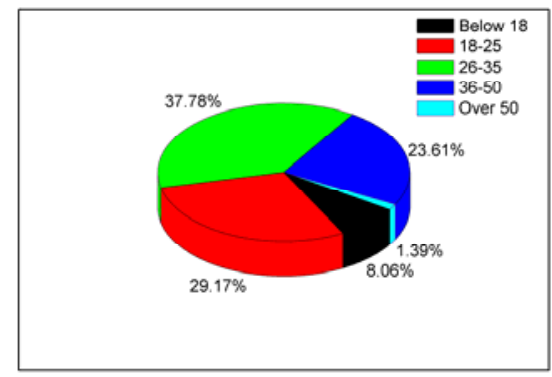

Figure. 2. User age structure

As shown in figure 2, the users are mainly young. At the age, the users of the social reading platform involved in this survey are mainly young people between the ages of 18 and 35, accounting for $67 \%$ of the total number. The number of users under 18 and over 50 years old are 58 and 10 , respectively. The proportion of the total number is $8 \%$ and $1.4 \%$, respectively. At the age of 36-50, the number of users is 170 , accounting for $23.6 \%$ of the total number. The results of the tenth national survey showed that more than $90 \%$ of adults with digital reading behavior were under the age of 49 . Therefore, the results of the survey are in accordance with the results of the tenth national survey. The 
results also indicate that young people are more inclined to read and try and use new things more positively than those over the age of 50 .

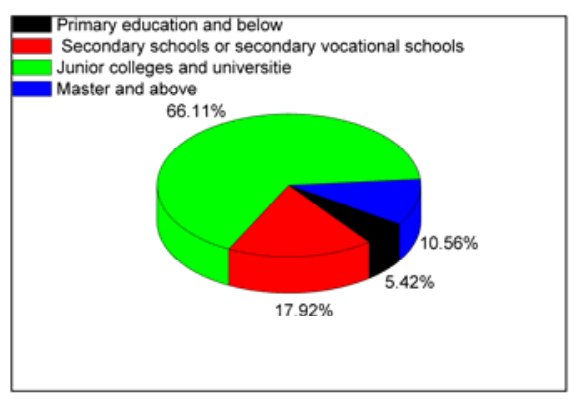

Figure. 3. User education level structure

The user education level is higher. The survey results show that the majority of social reading platform users belong to highly educated people. As shown in figure 3 , among respondents, $76.7 \%$ of the respondents have college education or above. Among them, there are 476 respondents with a college degree or bachelor degree, accounting for $66.1 \%$ of the total number. There are 76 respondents with master's degree or above, accounting for $10.6 \%$ of the total number. In addition, there are 129 respondents with qualifications in secondary schools or secondary vocational schools, accounting for $17.9 \%$ of the total number. There are 39 respondents with primary education and below, accounting for $5.4 \%$ of the total number. The results show that people with high academic qualification have high reading needs and are more willing to try new things.

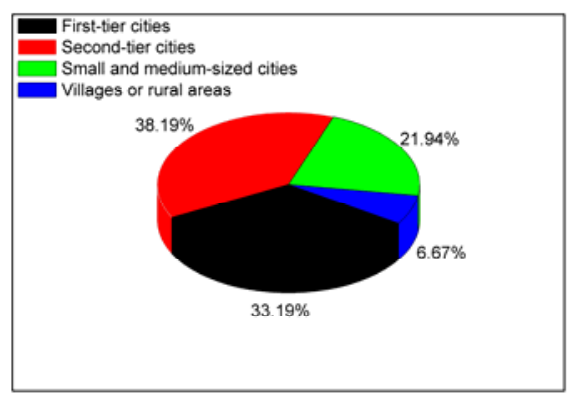

Figure. 4. The user's current residence structure

As shown in figure 4, the user is mainly located in the city. The statistical data show that the main body of the users of the social reading platform involved in this survey is urban residents, and the total ratio is more than $90 \%$ of the total number. Among them, the number of users living in Beijing, Shanghai and Guangzhou is 239 , accounting for $33.2 \%$ of the total respondents. The number of users living in second-tier cities, such as provincial capital, is 275 , accounting for $38.2 \%$ of the total respondents. The number of users living in small and medium-sized cities is 158 , accounting for $21.9 \%$ of the total respondents. There are only 48 people in rural or rural areas, accounting for $6.7 \%$ of the total number. This shows that the popularity rate of social reading platform in cities is higher than that in villages and towns. At the same time, this result is also consistent with the general rule of new media in China.

\subsection{Descriptive Analysis of Sample Use Behavior}

As shown in figure 5, from the way of login, the user is more inclined to use the social reading platform on the mobile device. Among the respondents, 663 users chose the mobile client as the login mode. 418 users chose the mobile phone to register, accounting for 58.1\%. 245 users chose tablet computers as the login mode and the ratio is $34 \%$. There are 313 users who choose a desktop or notebook computer, accounting for $43.5 \%$ of the total number. 


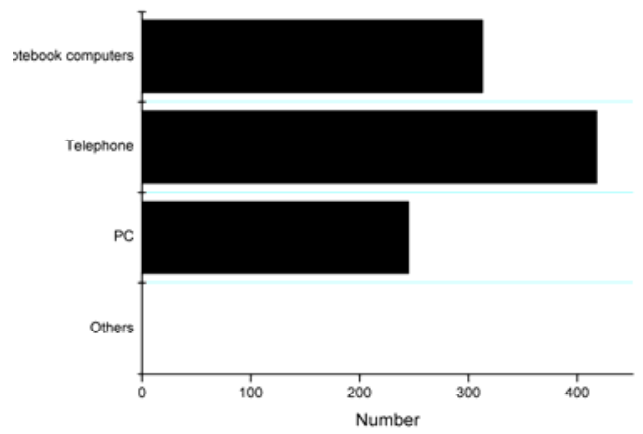

Figure. 5. The login mode of users using the socialized reading platform

According to the latest CNNIC data, in 2013, the proportion of netizens using mobile Internet rose to $81.0 \%$, up by $6.5 \%$. The proportion of netizens accessing the Internet through desktops and laptops decreased slightly. The above analysis is in line with the findings of CNNIC, indicating the arrival of mobile reading.

The registration time, use frequency and use time are important indicators of the user's use media. As shown in figure 6, from statistical data, the registration time of users involved in this survey is 487 within two years, accounting for nearly $70 \%$ of the total number, while there are 233 users over two years, accounting for $32.4 \%$ of the total number.

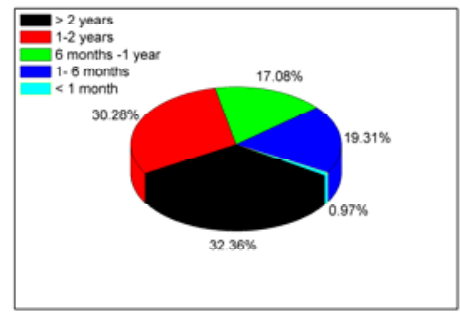

Figure. 6. The time situation of user registered social reading platform

\section{Conclusion}

First, to a certain extent, the demographic characteristics and basic use of socialized reading platform are understood. The social reading platform has the characteristics of high education, high income, urbanization and youth. The registration time of socialized reading platform is short, and the use time is more than half an hour each time. However, the use frequency is high. The usage habit is that the mobile terminal is first used as a login mode. Subscription and recommendation are the way to get information. In the content of reading, it is mainly to obtain news and information, followed by personalized information, such as emotional insight and entertainment jokes. Second, the author determines the factors that affect the social reading platform and the influence degree. The average value of the measurement items of social interaction factors is not high, and the average value of each measurement item of the reading cost is beyond the author's estimate. Third, it validates and quantifies the relationship between the use motivation and the use attitude. Among them, media defects will negatively impact the use of social reading platform. This conclusion has a vigilant effect on the development of social reading platform.

\section{References}

[1]. Sun, L., \& Xie, J. (2017). An exploration of reading promotion and readers' advisory at chinese public libraries. Reference \& User Services Quarterly, 56(2), 96.

[2]. Garner, R. (2018). Metacognition and reading comprehension. cognition and literacy. International Review of Education, 17(1), 11-26. 
[3]. Fowler, J. W., \& Peterson, P. L. (2016). Increasing reading persistence and altering attributional style of learned helpless children. Journal of Educational Psychology, 73(2), 251-260.

[4]. Mary, K. B. B. A. (2016). Proust and the squid: the story and science of the reading brain. Journal of the American Academy of Child \& Adolescent Psychiatry, 36(12), 1202-1203.

[5]. Ricci, G., Cipriani, F., Cuellogarcia, C. A., Brożek, J. L., Fiocchi, A., \& Pawankar, R., et al. (2016). A c linical reading on "world allergy organization-mcmaster university guidelines for allergic disease prevention (glad-p): probiotics". World Allergy Organization Journal, 9(1), 9.

[6]. Del, R. O. G. M., Espinel, A. I., \& Rosquete, R. G. (2016). Remedial interventions for children with reading disabilities: speech perception--an effective component in phonological training. Journal of Learning Disabilities, 35(35), 334-342.

[7]. Dean, M. D. (2016). A call to embrace social reading in higher education. Innovations in Education \& Teaching International, 53(3), 296-305.

[8]. Vlieghe, J., Vandermeersche, G., \& Soetaert, R. (2016). Social media in literacy education: exploring social reading with pre-service teachers. New Media \& Society, 18(5), 800-816. 